\title{
Identification of a Novel Human Peroxisomal 2,4-Dienoyl-CoA Reductase Related Protein Using the M13 Phage Protein VI Phage Display Technology
}

\author{
L. Amery ${ }^{1}$, G. P. Mannaerts ${ }^{1}$, S. Subramani ${ }^{2}$, P. P. Van Veldhoven ${ }^{1}$ and M. Fransen ${ }^{1 *}$ \\ ${ }^{1}$ Katholieke Universiteit Leuven, Faculteit Geneeskunde, Campus Gasthuisberg (O/N), Departement Moleculaire \\ Celbiologie, Herestraat 49, B-3000 Leuven, Belgium \\ ${ }^{2}$ University of California at San Diego, Department of Biology, 9500 Gilman Drive, La Jolla, CA 92093-0322, \\ USA
}

\begin{abstract}
Recently, we reported the successful use of the $g V I$-cDNA phage display technology to clone cDNAs coding for novel peroxisomal enzymes by affinity selection using immobilized antisera directed against peroxisomal subfractions (Fransen, M.; Van Veldhoven, P.P.; Subramani, S. Biochem. J., 1999, 340, 561-568). To identify other unknown peroxisomal enzymes, we further exploited this promising approach. Here we report the isolation and cloning of another novel human cDNA encoding a protein ending in the tripeptide AKL, a Cterminal peroxisomal targeting signal (PTS1). Primary structure analysis revealed that this molecule shared the highest sequence similarity to members of the 2,4-dienoyl-CoA reductase (DCR) family. However, functional analysis indicated that a recombinantly expressed version of the novel protein did not possess DCR activity with either 2-trans,4-trans-hexadienoyl-CoA or 2-trans,4-trans-decadienoyl-CoA as a substrate. The recombinant protein interacted with HsPex 5p, the human PTS1-binding protein. Binding was competitively inhibited by a PTS1-containing peptide and was abolished when the last amino acid of the PTS1 signal was deleted. Transfection of mammalian cells with gene fusions between green fluorescent protein (GFP) and the human cDNA confirmed a peroxisomal localization and, therefore, the functionality of the PTS1. These results further demonstrate the suitability of the $g V I$-cDNA phage display technology for cDNA expression cloning using an antibody as a probe.
\end{abstract}

\section{INTRODUCTION}

Peroxisomes are ubiquitous organelles of eukaryotic cells that carry out a large variety of metabolic functions. In mammals, these functions include the $\beta$-oxidation of diverse lipophilic carboxylates (e.g. very long chain fatty acids, 2methyl-branched fatty acids, and eicosanoids), the conversion of cholesterol into bile acids via $\beta$-oxidation, the initial degradation of 3-methyl-branched fatty acids via $\alpha$ oxidation, the synthesis of cholesterol, dolichol and other isoprenoids, the catalysis of the committed step in ether phospholipid synthesis, and the catabolism of certain purines, polyamines, amino acids, and peroxides [1,2]. The importance of peroxisomes to cellular metabolism is most clearly demonstrated by the occurrence of at least twenty-five human peroxisomal disorders [3]. Currently, more than forty different human peroxisomal proteins have been deposited in the SWISS-PROT/TrEMBL protein databases. The identification and characterization of these proteins at the molecular level have been of great value for our understanding of peroxisome function and for the identification of the molecular defects present in patients suffering from peroxisomal disorders. In this context, a more

\footnotetext{
*Address correspondence to this author at the Afdeling Farmacologie, Departement Moleculaire Celbiologie, Campus Gasthuisberg (O/N), Katholieke Universiteit Leuven, Herestraat 49, B-3000 Leuven, Belgium Tel: 32-16-345786; Fax: 32-16-345699; E-mail: marc.fransen@med. kuleuven.ac.be
}

complete inventory of peroxisomal proteins is of utmost importance.

Recently, we reported that the protein VI-phage display system provides a powerful selection method for the isolation of novel peroxisomal proteins from actual cDNA libraries [4]. The $g V I$-fusion expression cloning system, developed by Jespers et al. [5], is based on the fact that proteins can be displayed on filamentous phage through their attachment to the C-terminus of the minor coat protein VI, which is surface exposed [6]. The advantage of this system is that the display of proteins encoded by eukaryotic cDNAs, fused to the 3'-end of $g V I$, is not hampered by the presence of translational stop codons at the 3'-end of the cDNA and the lack of a suitable ribosome-binding site for prokaryotic expression at the 5'-end. Jespers et al. [5] first demonstrated the utility of this technology by subjecting an Ancylostoma caninum $\mathrm{g} V I$-cDNA library to biopanning against the serine proteases trypsin and human coagulation factor $\mathrm{Xa}$, and identified two novel serine protease inhibitors. More recently, Hufton et al. [7] successfully isolated from a $g V I-$ colorectal cancer library cDNAs encoding $\beta 2$-microglobulin and the constant regions of human immunoglobulins by biopanning with appropriate antibodies.

In this report, we expand and elaborate on our previous efforts to identify novel peroxisomal proteins using the pVIphage display technology [4] and report on the identification of a novel human peroxisomal 2,4-dienoyl-CoA reductase 
related protein (DCR-RP). These results serve to further highlight the promise of using the pVI-phage display technology for the high throughput screening of cDNA libraries.

\section{MATERIALS AND METHODS}

\section{Animals, Cell Lines and Strains}

White New Zealand rabbits were maintained on a standard laboratory chow diet. Animal care approval was granted by the local Institutional Ethics Committee. $\mathrm{CHO}$ cells were cultured at $37^{\circ} \mathrm{C}$ in MEM alpha medium supplemented with $10 \%(\mathrm{v} / \mathrm{v})$ fetal calf serum in a humified incubator $\left(5 \% \mathrm{CO}_{2}\right)$. To generate a cell line expressing HsPex14p-EGFP, CHO cells were transfected with pMF120 using the cationic polymer polyethylenimine [8] and stable clones were selected in the presence of $400 \mu \mathrm{g} / \mathrm{ml} \mathrm{G} 418$ sulphate (Across Organics). To construct pMF120, the cDNA insert of pMF34 [9] was amplified by PCR (Pfu DNA polymerase, primers HsPex14.3 and HsPex14.4 (Table 1)) and the resulting PCR product was gel purified, digested with $B g l$ II and Pst I and subcloned into the mammalian expression vector pEGFP-N1 (Clontech). The E. coli strain Top10F' (Invitrogen) was used for all transformations and plasmid isolations, the rescue of the phagemid libraries, and the expression and purification of recombinant proteins. To express recombinant proteins in $S$. cerevisiae, the yeast strain CB80 (MATa, leu2-1, ura3-52, his3-200, trp1-63) [10] was transformed with plasmids derived from pJR233 [11].

\section{Antibodies}

The bait antiserum Ab-MF16 was described previously [4]. The antiserum to HsPex $14 \mathrm{p}$ was raised in rabbits, as described for Ab-MF3 [9], using recombinant (His) 6- $^{-}$ HsPex14p [9] as antigen. The mouse 6xHis monoclonal antibody and the sheep anti-rabbit-Cy3 polyclonal antibody were obtained from Invitrogen and Sigma, respectively.

\section{Panning Procedure and Analysis of Selected Clones}

The rescue of the rat liver $g V I$-cDNA phagemid libraries, subcloned in the phagemid vectors pG6A, pG6B and pG6C [5], as well as the selection of $g V I-c D N A$ phage libraries by immunoaffinity was done exactly as described previously [4]. The insert size of the selected clones was determined by PCR-colony screening with the primers pG6f and pG6r. DNA sequencing of different cDNA inserts was performed by the method of Sanger [12] using vector- and gene-specific primers. Database searches and protein alignments were performed using the BLAST algorithm [13] and the multiple sequence alignment with hierarchical clustering [14], respectively. Searches for protein motifs were done with the Expasy ScanProsit tool (Swiss Institute of Bioinformatics).

\section{Cloning of the Full-Length Human DCR-RP cDNA}

Probing the EST database (dbEST) with the obtained rat cDNA sequence allowed us to compose a putative full-length

Table 1. List of Oligonucleotides (Restriction Sites are Underlined)

\begin{tabular}{|c|c|}
\hline Name & Sequence \\
\hline DCRMF1 & 5'-CACGGATCCAACATGACCCAGCAGCCGCC-3' \\
\hline DCRMR1 & 5'-GCTCTGCATGCACTACAGCTTAGCAGAGG-3' \\
\hline HsPex 14.3 & 5'-GGGA \\
\hline HsPex14.4 & 5'-AATCTGCAGGTCCCGCTCACTCTCGTT-3' \\
\hline pBADTOPO-F & 5'-GCTATGCCATAGCATTTTTATCC-3' \\
\hline pBADTOPO-R & 5'-GATTTAATCTGTATCAGGCTG-3' \\
\hline pG6f & 5'-CTCCCGTCTAATGCGCTTCCCTG-3' \\
\hline pG6r & 5'-GCTGCAAGGCGATTAAGTTGGGT-3' \\
\hline RedBglf1 & 5'-GCTCAGATCTGCAGGGATGGCCTCCTGGG-3' \\
\hline RedEcor 1 & 5'-GCTCGGAATTCTCAGAGCTTAGCTTTCTC-3' \\
\hline RedEcor2 & 5'-CCTTGGAATTCACTTAGCTTTCTCCTTAAA-3' \\
\hline RedLF.1 & 5'-GCAGGGATGGCCTCCTGGG-3' \\
\hline RedLF2.F & 5'-CCTGCTGAACACATCAGTTC-3' \\
\hline RedLR.1 & 5'-GCTCAGAGCTTAGCTTTCTCC-3' \\
\hline RedLR2.F & 5'-CAGAGGAGACCTCCTCAGG-3' \\
\hline RedSphr1 & 5'-GCTCGGCATGCCTCAGAGCTTAGCTTTCTC-3' \\
\hline
\end{tabular}


mouse open reading frame (accession no. AF242204). Using this composed mouse cDNA sequence as a query, we screened the human dbEST. An alignment of the obtained ESTs resulted in a non-contiguous cDNA sequence encoding the putative start (accession numbers AA232176, AA232159 and H84466) and stop codon (accession nos. AI435448, AI458176 and AI268603) of the human DCR-RP open reading frame. The complete coding sequence of this human cDNA was obtained by RT-PCR. In a first step, human liver cDNA was generated as described by Wylin et al. [15]. In a subsequent step, 2 sequential PCR reactions were performed [first reaction: $1 \mu \mathrm{l}$ of the human liver cDNA as template, gene-specific primers RedLF.1 and RedLR.1 (Table 1), Pfu polymerase (Stratagene); second reaction: $1 \mu \mathrm{l}$ of the first PCR reaction (diluted 1:100) as template, same set of primers, $P f u$-polymerase]. Direct cloning of the obtained PCR product into the TopoTA cloning vector (Invitrogen) was performed by the addition of a 3'-A overhang to the gelpurified PCR product as described by the manufacturer. The resulting cDNA insert of the construct, pLA40, was sequenced using the vector primers pBADTOPO-F and pBADTOPO-R, and the gene-specific primers RedLF2.F and RedLR2.F (Table 1).

\section{In Vitro Pex5p Binding Experiments}

In order to express a (His) $)_{6}$-tagged human DCR-RP with or without its putative PTS1, we amplified the cDNA insert of pLA40 by PCR using the primer sets RedBglf $1 / \operatorname{RedEcor} 1$ (for DCR-RP) and RedBglf1/ RedEcor2 (for DCR-RP $\mathrm{ALeu303}_{\text {) }}$ (Table 1). The obtained PCR fragments were gel purified, digested with $B g l$ II and $E c o$ RI, and subcloned into the $B g l$ II/Eco RI-digested pBADHisB expression vector (Invitrogen). Top10F' cells were transformed and correct clones were selected. To purify the (His) $)_{6}$-tagged proteins DCR-RP (encoded by pLA58) and DCR-RP ${ }_{\triangle \mathrm{Leu} 303}$ (encoded by pLA60), the expression of the recombinant proteins was induced with $0.2 \%(\mathrm{w} / \mathrm{v})$ arabinose. Four hours after induction, the (His) $)_{6}$-tagged fusion protein was purified under denaturing conditions (6 $\mathrm{M}$ guanidinium chloride) using Ni-NTA Agarose (Amersham Pharmacia Biotech) according to the manufacturer's instructions. Purified protein was subjected to SDS-PAGE and transferred to nitrocellulose by semi-dry blotting. Blots were incubated with bacterial lysates expressing biotinylated human Pex5p and further processed with streptavidin-alkaline phosphatase as described by Fransen et al. [16]. For the competition experiments, 1 $\mu \mathrm{g}$ of the recombinant (His) $)_{6}$-tagged fusion proteins was coated in wells of a 96-well microtiter plate and incubated with the biotinylated Pex5p fusion protein as described previously [17]. During the incubation with Pex5p, increasing amounts of the peptides pSKL (CSYHKHLKPLQSKL) or pUNSKL (CVHESYHKHLKPLQ) were added. Note that pUNSKL resembles pSKL, but does not contain the Cterminal tripeptide SKL [17].

\section{Transfections and Immunofluorescence}

To construct plasmids for the expression of EGFP-DCRRP (pLA59) and EGFP-DCR-RP ${ }_{\Delta^{\mathrm{Leu}} 303}$ (pLA61) in mammalian cells, the primer combinations RedBglf1/ RedEcor1 and RedBglf1/RedEcor2 (Table 1) were, together with the template pLA40, used to generate 2 PCR fragments. These PCR products were, after digestion with $B g l \mathrm{II}$ and $E c o$ RI, subcloned into the $B g l \mathrm{II} / E c o$ RI digested pEGFP-C1 vector (Clontech). Chinese hamster ovary (CHO) cells were transiently transfected using the polyethylenimine transfection method [8]. Immunofluorescence studies were essentially done as described [17]. Double immunofluorescence studies were performed using the rabbit anti-Pex $14 p$ antiserum or Ab-MF16 in conjunction with Cy3-conjugated sheep anti-rabbit IgGs (Sigma). Fluorescence was observed under a Leica DMR microscope equipped with standard FITC filters for GFP, and RITC filters for $\mathrm{Cy} 3$.

\section{Bacterial and Yeast Expression of Rat DCR and Human DCR-RP}

To compare the DCR activity of HsDCR-RP with the earlier described peroxisomal 2,4-dienoyl-CoA reductase [4], we transferred the HsDCR-RP cDNA into the PinPoint Xa3 expression vector (Promega). Therefore, the 1800 bp Bam HI/Hinc II fragment of pLA40 was subcloned into Xa3 digested with Bam HI and Sma I. In order to express the rat peroxisomal DCR in yeast, the cDNA insert of pMF45 [4] was amplified by PCR using the primer combination DCRMF1/DCRMR1 (Table 1). The resulting PCR fragment was digested with Bam $\mathrm{HI}$ and $S p h$ I and used to replace the Bam HI/Sph I-fragment of pJR233 (pLA113). To generate pLA114, a yeast expression plasmid encoding human DCR-RP, the cDNA encoding this protein was amplified by PCR (template pLA40, primer set RedBglf1/RedSphr1 (Table 1)), digested with $B g l \mathrm{II} / S p h \mathrm{I}$, and exchanged with the Bam HI/Sph I-fragment of pJR233. Yeast transformants were selected and grown on minimal medium containing $0.67 \%(\mathrm{w} / \mathrm{v})$ yeast nitrogen base without amino acids (Difco), $2 \%(\mathrm{w} / \mathrm{v})$ glucose and supplemented with bases and amino acids $(20-150 \mu \mathrm{g} / \mathrm{ml})$ as required (Sc-ura). Transformed yeast cells were grown at $30^{\circ} \mathrm{C}$ with shaking in selective medium with $0.5 \%(\mathrm{w} / \mathrm{v})$ glucose as sole carbon source until the glucose concentration was very low $(\sim 0.05$ $\%(\mathrm{w} / \mathrm{v})$, usually $12-16 \mathrm{~h})$. Cell extracts were prepared as follows: yeast cells corresponding to $50 \mathrm{~A}_{600}$ units (volume $\mathrm{x}$ cell density) were harvested by centrifugation and resuspended in $400 \mu \mathrm{l}$ of $50 \mathrm{mM}$ potassium phosphate $\mathrm{pH}$ 7.5. Cells were broken by vortexing for $5 \mathrm{~min}$ at $4^{\circ} \mathrm{C}$ in the presence of $200 \mu \mathrm{l}$ of glass beads $(500 \mu \mathrm{m})$. After centrifugation $\left(15,000 \mathrm{~g}, 10 \mathrm{~min} ; 4^{\circ} \mathrm{C}\right)$, the extracts were used for enzyme measurements.

\section{Synthesis of Substrates and 2,4-dienoyl-CoA Reductase Measurements}

2,4-Dienoyl-CoA reductase activities, using bacterial lysates and yeast lysates expressing no recombinant protein, rat peroxisomal DCR or human DCR-RP, were measured as described by Fransen et al. [4], using $30 \mu \mathrm{M}$ 2-trans,4trans-hexadienoyl-CoA (sorboyl-CoA) or 2-trans,4-trans- 
decadienoyl-CoA as substrate. The latter CoA-ester was prepared enzymatically from 2-trans,4-trans-decadienoic acid by means of acyl-CoA synthetase (Pseudomonas fragi; Boehringer), exactly as described by Fransen et al. [4] for the synthesis of sorboyl-CoA. After completion of the reaction, the reaction mixture was acidified to $\mathrm{pH} 4$, extracted with heptane and loaded on a $\mathrm{C}_{18}$-solid phase extraction column (500 mg; Bond Elut, Varian). After washing the column with water, the CoA-ester was eluted with methanol/water (1:1 by vol.). Spectral analysis of the CoA-ester, in $10 \mathrm{mM}$ K-phosphate buffer $\mathrm{pH} 7.0$, showed the expected absorbance maxima at 260 and $300 \mathrm{~nm}$ (ratio $\mathrm{A}_{300}$ to $\mathrm{A}_{260}$ 0.7). 2Trans,4-trans-decadienoic acid was prepared from 2-trans,4trans-decadienal (Aldrich) by $\mathrm{Ag}_{2} \mathrm{O}$ oxidation according to Thomason and Kubler [18] with some modifications and further purified by silica column chromatography using a gradient of heptane/diethylether (95:5 to 20:80 by vol.). Purity was monitored by thin layer chromatography (solvents: heptane/diethylether/acetic acid (60:40:1, by vol.) and chloroform/methanol/25\%(v/v) ammonia (60:40:5, by vol.)). Spectral analysis of the acid, dissolved in ethanol, showed the typical absorbance maximum at $251 \mathrm{~nm}$.

\section{RESULTS}

\section{Indirect Immunofluorescence Microscopy Demonstrates that Ab-MF16 Recognizes Peroxisomes}

We previously characterized a rabbit antiserum, AbMF16, that recognizes at least 25 different rat liver peroxisomal proteins in Western blot experiments, and reported its successful use to select $g V I$-fusion phages carrying cDNAs coding for novel peroxisomal proteins [4]. The remarkable specificity of this bait antiserum was further illustrated by indirect immunofluorescence microscopy with CHO cells expressing human Pex14p, a peroxisomal integral membrane protein [9], fused to EGFP. The distribution pattern of the proteins recognized by Ab-MF16 (Fig. 1A) was superimposable on the distribution pattern of HsPex 14pEGFP (Fig. 1B), demonstrating that the Ab-MF16-reactive proteins are indeed peroxisomal proteins.

\section{Affinity Selection of Ab-MF16-Reactive Fusion Phages}

Employing previously optimized conditions [4], we performed a biopanning experiment with Ab-MF16 using 3 rounds of selective enrichment. After the third panning round, the size of the cDNA inserts of 15 randomly picked clones was analyzed by PCR. Such analysis demonstrated that we had isolated two different clones. Repanning these clones against the bait Ab-MF16 resulted in 15- to 20-times more phage than panning against the corresponding preimmune serum, suggesting specificity of the isolated clones. Sequencing the cDNA inserts of both clones revealed that one encoded the peroxisomal 2,4-dienoyl-CoA reductase we previously isolated [4], and that the other encoded a novel cDNA (pVI-ARL) with a partial open reading frame of 215 amino acids ending in the tripeptide ARL. This C-terminal tripeptide is reportedly sufficient for peroxisomal targeting of passenger proteins $[11,19]$.
The pVI-ARL Clone Encodes A Novel 2,4-Dienoyl-CoA Reductase Related Protein

By probing the expressed sequence tag (EST) database with the novel partial rat cDNA, we delineated a mouse open reading frame (accession no. AF242204) encoding a putative protein of 303 amino acids with a calculated molecular mass of $32.4 \mathrm{kDa}$. Rescanning the human EST database using the composed mouse cDNA as a query, we deduced the 5'- and 3 '-end of a cDNA encoding a putative human homologue. The full-length human open reading frame (accession no. AJ250303), which was obtained by PCR, also encoded a protein of 303 amino acids with a calculated molecular mass similar to that of the mouse protein. Homology searches revealed that both the human and mouse proteins, which are $71.9 \%$ identical and end in AKL, shared the highest homology with members of the 2,4-dienoyl-CoA reductase (DCR) family. For example, the human protein is $35 \%$ identical to the mouse (accession no. NP_036063), rat (accession no. AAD02333) and yeast (accession no. NP_014197) peroxisomal 2,4-dienoyl-CoA reductases (Fig. 2), and $33 \%$ identical to the rat (accession no. S11021) and human (accession no. AAB09423) mitochondrial 2,4dienoyl-CoA reductases (data not shown). Based on this sequence homology, we named the novel protein a 2,4dienoyl-CoA reductase related protein (abbreviated to DCR$\mathrm{RP}$ ). During the preparation of this manuscript, the fulllength rat DCR-RP (Fig. 2) was released in the database (accession nos. AAD38447 and AAF14047). Note that all
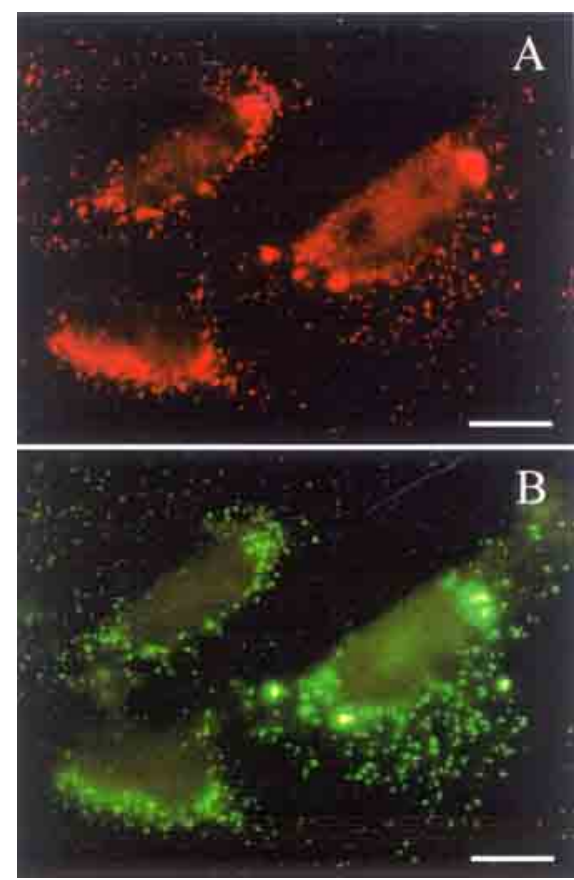

Fig. (1). Ab-MF16 recognizes peroxisomes by immunofluorescence microscopy. Double localization of Ab-MF16reactive proteins (A) and HsPex14p-EGFP (B) in CHO cells clearly demonstrates that the cell organelles marked with AbMF16 (RITC filter) can be identified as peroxisomes by superimposition on the appropriate HsPex14p-EGFP (FITC filter) positive particles. Scale bar $=10 \mu \mathrm{m}$. 


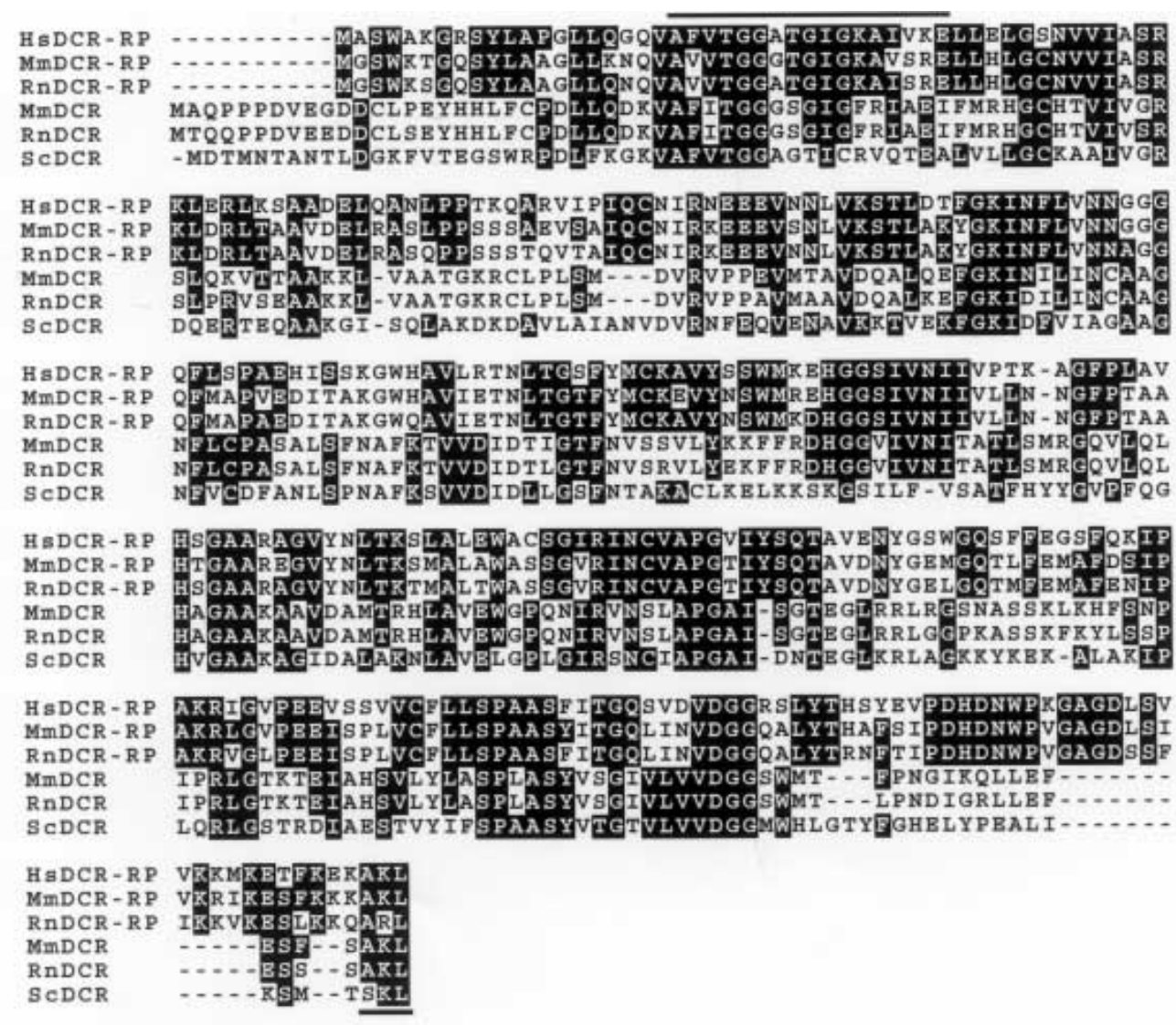

Fig. (2). Amino acid alignment of the peroxisomal members of the DCR protein family. Human (accession no. AJ250303), mouse (accession no. AF242204) and rat (accession no. AAD02333) DCR-RP were aligned with the other peroxisomal members of the DCR protein family (accession nos. NP_036063, AAF14047 and S50729 for mouse, rat and yeast DCR, respectively) using the multiple sequence alignment with hierarchical clustering [14]. Amino acids identical in 3 of 6 sequences were depicted in reverse type. The putative nucleotide-binding sequences [27] are indicated by an overline.

experiments reported below were performed using the fulllength human DCR-RP.

\section{DCR-RP Localizes to Peroxisomes and Contains a Functional C-Terminal Peroxisomal Targeting Signal}

To confirm the peroxisomal localization of human DCR$\mathrm{RP}$, we expressed the protein in $\mathrm{CHO}$ cells as a C-terminal fusion to the heterologous reporter protein EGFP. The experiment demonstrated that the obtained punctate EGFPDCR-RP fluorescence pattern (Fig. 3A) co-localized with Pex $14 p$, a peroxisomal integral membrane protein (Fig. 3B). To determine if EGFP-DCR-RP was indeed targeted to the peroxisomes by its C-terminal tripeptide AKL, we generated a construct encoding a fusion protein, EGFP-DCR-RP ${ }_{\Delta \text { Leu303, }}$, in which the extreme C-terminal amino acid was deleted. Transfecting $\mathrm{CHO}$ cells with this construct resulted in a cytosolic fluorescence pattern (Fig. 3C). To further evaluate the role of the C-terminal tripeptide AKL, we expressed (His) -tagged versions of DCR-RP and DCR-RP $_{\triangle \text { Leu303 }}$ and analyzed the interaction of those molecules with HsPex $5 p$, the human import receptor for peroxisomal proteins containing a C-terminal targeting sequence [17]. By using a blot overlay assay [16], an interaction between DCR-RP and Pex5p (Fig. 4A, lane 2), but not between DCR-RP ${ }_{\Delta \text { Leu303 }}$ (Fig. 4A, lane 1) and Pex 5p, was apparent. Both proteins were present at equal levels (Fig. 4B). In addition, as shown in Fig. 4C), HsPex5p was able to bind to (His) ${ }_{6}$-DCR-RP coated in microtiter plate wells and this interaction was competitively inhibited by a peptide ending in SKL, but not by a related peptide lacking this PTS1 sequence.

\section{Recombinant Human DCR-RP has no DCR Activity}

2,4-Dienoyl-CoA reductases are required for the complete $\beta$-oxidation of unsaturated fatty acids having double bonds in even- or odd-numbered positions [20-22]. In a search for the function of DCR-RP in peroxisomal metabolism, we tested whether or not human DCR-RP possessed DCR activity. We expressed the molecule in $E$. coli as a biotinylated fusion protein and assayed its activity on 2trans,4-trans-hexadienoyl-CoA and 2-trans,4-transdecadienoyl-CoA. As a positive control, we simultaneously expressed the biotinylated peroxisomal 2,4-dienoyl-CoA reductase [4]. Although both biotinylated proteins were expressed equally, and similar amounts of both proteins were 

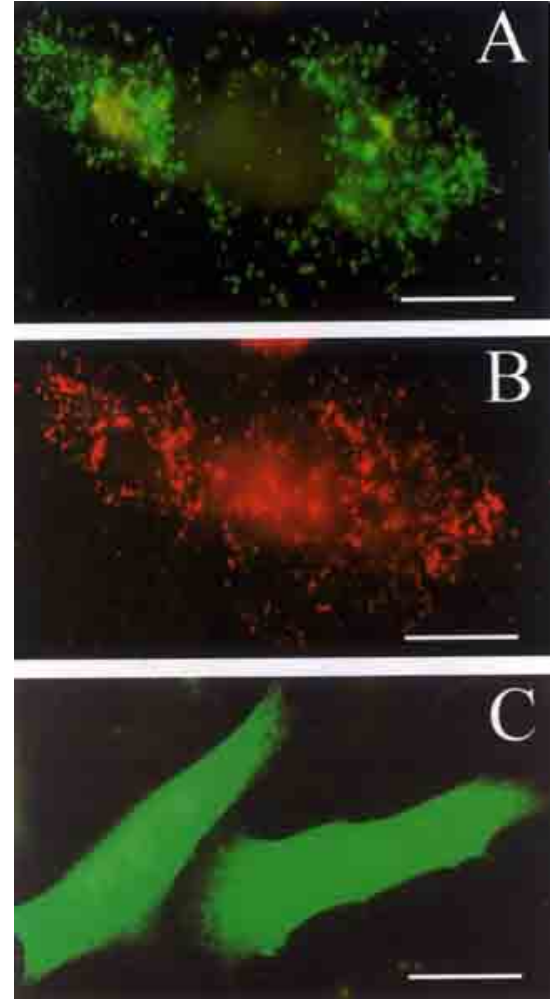

Fig. (3). Human DCR-RP is a peroxisomal protein. CHO cells were transfected with pEGFP-C1 derivatives encoding EGFP$\operatorname{DCR}-R P(\mathbf{A}, \mathbf{B})$ and EGFP-DCR-RP ${ }_{\triangle \mathrm{Leu} 303}(\mathbf{C})$. Two days after transfection the cells were processed for immunofluorescence. Double localization studies of EGFP-DCR-RP (FITC filter) (A) and Pex14p (RITC filter) (B) demonstrated that the cell organelles labeled with the EGFP-fusions were peroxisomes. Cells expressing EGFP-DCR-RP ${ }_{\triangle \mathrm{Leu} 303}$ (C) gave rise to a cytosolic staining pattern. Scale bar $=10 \mu \mathrm{m}$.

recovered in the soluble fraction after sonication of the bacteria (data not shown), only the lysates obtained from the

A

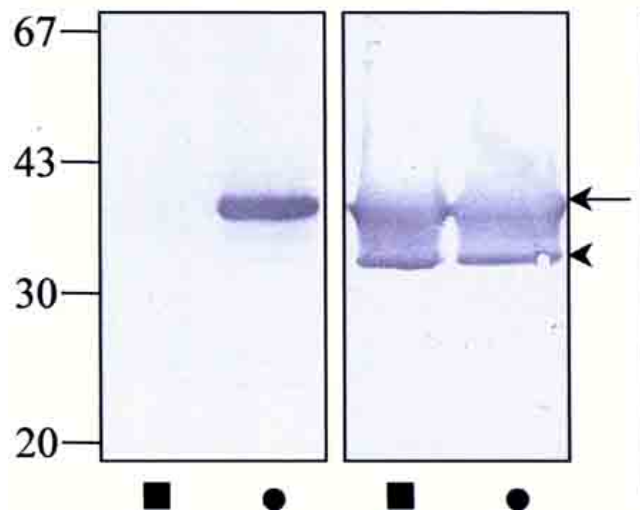

positive control processed the substrates (Table 2). Similar results were obtained when DCR-RP and the positive control were expressed in the budding yeast $S$. cerevisiae (Table 2).

\section{DISCUSSION}

Previously, we demonstrated that the $g V I$-based phage display technology provides an effective and straightforward means of cloning cDNAs encoding novel peroxisomal proteins using antisera raised against peroxisomal subfractions [4]. Here, we further exploit this in vitro selection system and report on the molecular identification of a novel peroxisomal protein which shares high similarity to members of the DCR protein family. Despite the sequence homology, we were unable to demonstrate that this protein, which we named DCR-RP, has DCR activity in vitro. In contrast to the peroxisomal 2,4-dienoyl-CoA reductase we previously characterized [4], DCR-RP, expressed in either bacteria or yeast, was not active on substrates commonly used for such analysis (2-trans,4-trans-hexadienoyl-CoA $\left(\mathrm{C}_{6}\right.$-dienoyl-CoA $)$ and 2-trans,4-trans-decadienoyl-CoA $\left(\mathrm{C}_{10^{-}}\right.$ dienoyl-CoA)). We do know that the human DCR-RP is expressed properly in $E$. coli because a protein of the right size is generated and it has the authentic C-terminal peptide that is recognized by human Pex5p. Currently, it is not clear whether or not (i) DCR-RP is a 2,4-dienoyl-CoA reductase at all, (ii) the expression of DCR-RP in E. coli as well as in $S$. cerevisiae yielded an inactive protein or (iii) the physiological substrate for DCR-RP differs from the tested $\mathrm{C}_{6^{-}}$or $\mathrm{C}_{10^{-}}$dienoyl-CoA substrates. With respect to the latter point, it has been suggested that 2,4-dienoyl-CoA reductase may be involved in the chain-shortening of some eicosanoids (e.g. leukotrienes B4 and E4) [23,24]. However, the proper intermediates to test this hypothesis are currently not available.

Although the biological role of DCR-RP remains a fundamental question to be addressed, our results firmly establish that human DCR-RP is a peroxisomal protein,

C

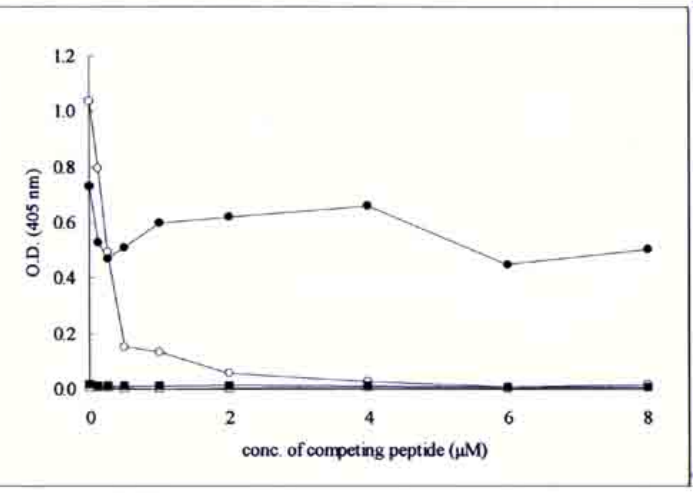

Fig. (4). In vitro binding of human DCR-RP to the PTS1 receptor Pex5p. One $\mu \mathrm{g}$ of bacterially expressed, purified (His) ${ }_{6}$-DCR-RP (-) or (His) ${ }_{6}$-DCR-RP ${ }_{\Delta \mathrm{Leu} 303}(\mathbf{\square})$ was subjected to SDS-PAGE and tranferred to nitrocellulose (A, B) or coated into a microtiter well (C). The molecules immobilized on the membrane were probed with a bacterial lysate containing biotinylated Pex 5p (A) or a mouse anti-His antibody (B) and complexes were visualized as previously described [16]. The migration of the molecular mass markers (given in $\mathrm{kDa}$ ) is shown at the left side. The protein band marked with a small arrowhead most likely represents a proteolytic degradation product of the (His) $)_{6}$-tagged HsDCR-RP fusion proteins (arrow). The molecules immobilized in the microtiter wells (C) were incubated with lysates expressing biotinylated Pex5p in the presence of the indicated amounts of the peptides pSKL $(\mathbf{O}, \square)$ or pUNSKL $(\boldsymbol{O}, \boldsymbol{\square})$. pUNSKL resembles pSKL, but does not contain the C-terminal tripeptide SKL [17]. 
Table 2. Recombinant HsDCR-RP has no DCR Activity. Bacterial and Yeast Lysates Expressing no Recombinant Protein (-), Human DCR-RP or rat DCR were Analyzed for 2,4-Dienoyl-CoA Reductase Activity as described in the 'Materials and Methods' Section. The Results of a Representative Experiment, Values Expressed in mU/mg of Protein, are Shown

\begin{tabular}{|c|c|c|c|}
\hline Host & & 2-trans,4-trans-hexadienoyl-CoA & 2-trans,4-trans-decadienoyl-CoA \\
\hline \hline E. coli & - & 3.6 & 5.6 \\
\hline & DCR-RP & 3.2 & 4.8 \\
\hline & DCR & 20.7 & 61.7 \\
\hline S. cerevisiae & - & 3.6 & 3.5 \\
\hline & DCR-RP & 3.4 & 3.0 \\
\hline & DCR & 80.5 & 416.9 \\
\hline
\end{tabular}

directed to the organelle by a C-terminal peroxisomal targeting signal. That is, (i) DCR-RP, but not DCR$\mathrm{RP}_{\triangle \mathrm{Leu} 303}$, bound specifically to Pex5p, the import receptor for peroxisomal matrix proteins with a C-terminal peroxisomal targeting signal and (ii) transfection of $\mathrm{CHO}$ cells with EGFP-DCR-RP, but not EGFP-DCR-RP ${ }_{\triangle \mathrm{Leu} 303}$, resulted in a fluorescent staining pattern coincident with that of the peroxisomal marker protein Pex 14p. Although these experiments were performed using human DCR-RP, similar results are predicted for the rat homologue. Indeed, the rat cDNA encoding the $215 \mathrm{C}$-terminal amino acids of rat DCR$\mathrm{RP}$ was isolated by biopanning against Ab-MF16, an antiserum raised against rat peroxisomal proteins [4]. In addition, ARL, the C-terminal tripeptide of the rat protein appears sufficient for peroxisomal targeting of passenger proteins $[11,19]$.

Although display on filamentous phage is a well established tool for peptide and protein engineering, the use of this technology for the display and selection of high diversity cDNA libraries is not yet widespread. While it is known that phage display indeed has certain intrinsic limitations (e.g. the use of a bacterial host may preclude the correct folding or post-translational modifications of some proteins; proteins need to be secreted from E. coli), the most likely explanation for the absence of more universal acceptance is that in the classical pIII and pVIII phage display systems, cDNAs with indeterminate 5 '- and 3 '-ends and potential translational stops are not straightforward to display. That is, cDNAs have to be inserted between a periplasmic export leader sequence and the $\mathrm{N}$-terminus of the phage minor or major coat proteins pIII or pVIII, matching the two reading frames [25]. Direct fusion of proteins to the C-terminus of pIII or pVIII will hamper phage assembly.

To circumvent these problems, alternative display systems have been developed. Besides the pVI-phage display technology, the best known alternative method is the pJuFo system [26]. This system involves the fusion of pIII with the leucine zipper of c-Jun while the translated cDNA products are fused to the C-terminal end of the leucine zipper of cFos. Upon phage assembly, the zipper domains associate at the tip of the phage. Experimental data show that the display levels of various proteins, when fused to pVI, are generally lower than when fused via the c-Jun-c-fos heterodimer to pIII
[5,7]. In case of a variant of the atrial natriuretic peptide [5] and the peroxisomal membrane protein Pex $3 p$ (Fransen M. and Subramani S., unpublished data), only $0.004 \%$ and $0.01 \%$ of the phage clones, respectively, display the fusion protein. Whether this low incorporation level simply reflects a differential expression level of the recombinant fusion proteins in comparison with their helper phage encoded counterparts, or that the anchorage of foreign sequences to the C-terminus interferes with the incorporation of $\mathrm{pVI}$ during phage morphogenesis, is not clear [5]. Notice that although the interference of $\mathrm{pVI}$-fusion proteins with the incorporation of $\mathrm{pVI}$ during phage morphogeneses may introduce a bias between different proteins for efficient display on phage, all the proteins we tested could be expressed on the phage coat as a pVI-fusion protein. The observation that different classes of proteins (e.g. protease inhibitors, peroxisomal proteins, immunoglobulins, and $E$. coli alkaline phosphatase) can be displayed on the phage as pVI-fusion proteins, further illustrates the general applicability of this technology $[4,5,7]$. In addition, the low incorporation level of these $\mathrm{pVI}$-fusion proteins does not seem to preclude the (immuno)affinity selection of the pVIfusion phages. However, previous experiments with a mixture of specific clones showed that subsequent panning rounds select for clones with expression advantages that will dominate the entire output [4]. Finally, the gVI phagemid vectors appear, in comparison with the pJuFo vector, to be less prone for deletions as the selection rounds add up [7].

Despite the fact that the pVI-phage display technology allows the rapid isolation of rare fusion phage clones from complex libraries with only minute amounts of bait, this system has not taken off as one would expect for a robust system. Nevertheless, our previous [4] and current results (above), as well as the results recently obtained by Hufton $e t$ $a l$. [7], further establish that the $g V I$-based phagemid vectors should be widely applicable for the rapid selection of cDNAs encoding immunogenic ligands.

\section{ACKNOWLEDGEMENTS}

The authors are grateful to Dr. L. Jespers and the Center for Transgene Technology and Gene Therapy (Leuven, Belgium) for the phagemid vectors pG6A, pG6B and pG6C 
and to Dr. A. Hartig (Vienna, Austria) for the yeast strain CB80 and the plasmid pJR233. The excellent technical support of C. Brees, J. Van Looy and V. Brys is greatly appreciated. L.A. is supported by a fellowship from the 'Vlaams Instituut voor de Bevordering van het Wetenschappelijke-Technologisch Onderzoek in de Industrie (IWT)'. M.F. is a postdoctoral fellow of the 'Fonds voor Wetenschappelijk Onderzoek - Vlaanderen (FWO)'. This work is supported by a 'Geconcerteerde Onderzoeksacties' grant (GOA/99/09) from the Flemish government and by grant FWO G.0204.99. S. Subramani was funded by grant NIH DK41737.

\section{ADDENDUM IN PROOF}

While this manuscript was in review, Das et al. (J. Biol. Chem., 2000, 275, 24333-24340) reported the cloning of a peroxisomal trans-2-enoyl-CoA reductase which was identical to DCR-RP, and indeed, did not possess 2,4dienoyl-CoA-reductase activity.

\section{LIST OF ABBREVIATIONS}

$\begin{array}{ll}\mathrm{CHO} & =\text { Chinese hamster ovary } \\ \mathrm{dbEST} & =\text { EST database } \\ \mathrm{DCR} & =2,4-\text { Dienoyl-CoA reductase } \\ \mathrm{DCR}-\mathrm{RP} & =\text { (Enhanced) Green fluorescent protein } \\ (\mathrm{E}) \mathrm{GFP} & =\text { Expressed sequence tag } \\ \mathrm{EST} & =\mathrm{M} 13 \text { gene encoding protein VI } \\ \text { gVI } & =\mathrm{C}-\text { Terminal peroxisomal targeting signal } \\ \mathrm{PTS} 1 & =\mathrm{M} 13 \text { phage protein } \mathrm{VI}\end{array}$

Sequence data reported in this paper have been deposited with the EMBL/Genbank Data Libraries (accession numbers $\mathrm{AJ} 250303$ and AF242204 for the human and mouse peroxisomal 2,4-dienoyl-CoA reductase related protein, respectively).

\section{REFERENCES}

[1] Reddy, J.K.; Mannaerts, G.P. Annu. Rev. Nutr., 1994, 14, 343-370.

[2] Mannaerts, G.P.; Van Veldhoven, P.P.; Casteels, M. Cell Biochem. Biophys., 1999, 31, 321-335.

[3] Moser, H. Front. Biosci., 2000, 5, D307-D311.

[4] Fransen, M.; Van Veldhoven, P.P.; Subramani, S. Biochem. J., 1999, 340, 561-568.

[5] Jespers, L.S.; Messens, J. H.; De Keyser, A.; Eeckhout, D.; Van Den Brande, I.; Gansemans, Y.G.; Lauwereys, M.J.;
Vlasuk, G.P.; Stanssens, P.E. Bio/Technology, 1995, 13, 378-382.

[6] Makowski, L. J. Mol. Biol., 1992, 228, 885-892.

[7] Hufton, S.E.; Moekerk, P.J.; Meulemans, E.; de Bruine, A.; Arends, J.-W.; Hoogenboom, H.R. J. Immunol. Methods, 1999, 231, 39-51.

[8] Boussif, O.; Lezoualc'h, F.; Zanta, M.A.; Mergny, M.; Sherman, D.; Demeneix, B.; Behr, J.-P. Proc. Natl. Acad. Sci. USA, 1995, 92, 7297-7303.

[9] Fransen, M.; Terlecky, S.R.; Subramani, S. Proc. Natl. Acad. Sci. USA, 1998, 95, 8087-8092.

[10] Brocard, C.; Lametschwandtner, G.; Koudelka, R.; Hartig, A. EMBO J., 1997, 16, 5491-5500.

[11] Lametschwandtner, G.; Brocard, C.; Fransen, M.; Van Veldhoven, P.P.; Berger, J.; Hartig, A. J. Biol. Chem., 1998, 273, 33635-33643.

[12] Sanger, F.; Nicklen, S.; Coulson, A.R. Proc. Natl. Acad. Sci. USA, 1977, 74, 5463-5467.

[13] Altschul, S.F.; Madden, T.L.; Schäffer, A.A.; Zhang, J.; Zhang, Z.; Miller, W.; Lipman, D.J. Nucleic Acids Res., 1997, 25, 3389-3402.

[14] Corpet, F. Nucleic Acids Res., 1988, 16, 10881-10890.

[15] Wylin, T.; Baes, M.; Brees, C.; Mannaerts, G.P.; Fransen, M.; Van Veldhoven, P.P. Eur. J. Biochem., 1998, 258, 332-338.

[16] Fransen, M.; Brees, C.; Van Veldhoven, P.P.; Mannaerts, G.P. Anal. Biochem., 1996, 242, 26-30.

[17] Fransen, M.; Brees, C.; Baumgart, E.; Vanhooren, J.C.T.; Baes, M.; Mannaerts, G.P.; Van Veldhoven, P.P. J. Biol. Chem., 1995, 270, 7731-7736.

[18] Thomason S.C.; Kubler D.G. J. Chem. Educ., 1968, 45, 546-547.

[19] Gould, S.J.; Keller, G.-A.; Hosken, N.; Wilkinson, J.; Subramani, S. J. Cell Biol., 1989, 108, 1657-1664.

[20] Dommes, V.; Baumgart, C.; Kunau, W.-H. J. Biol. Chem., 1981, 256, 8259-8262.

[21] Smeland, T.E.; Nada, M.; Cuebas, D.; Schulz, H. Proc. Natl. Acad. Sci. USA, 1992, 89, 6673-6677.

[22] He, X.-Y.; Shoukry, K.; Chu, C.; Yang, J.; Sprecher, H.; Schulz, H. Biochim. Biophys. Res. Commun., 1995, 215 , 15-22.

[23] Shirley, M.A.; Murphy, R.C. J. Biol. Chem., 1990, 265, 16288-16295.

[24] Sala, A.; Voelkel, N.; Maclouf, J.; Murphy, R.C. J. Biol. Chem., 1990, 265, 21771-21778.

[25] Light, J.; Maki, R.; Assa-Munt, N. Nucleic Acids Res., 1996, 24, 4367-4368

[26] Crameri, R.; Suter, M. Gene, 1993, 137, 69-75.

[27] Wierenga, R.K.; De Mayer, M.C.H.; Hol, W.G.J. Biochemistry, 1985, 24, 1357-1363. 